\title{
Groupement de recherche européen (GdRE) du CNRS. Terres cuites architecturales et nouvelles méthodes de datation (TCA médiévales et datation)
}

Christian Sapin et Pierre Guibert

\section{(2) OpenEdition Journals \\ Édition électronique \\ URL : https://journals.openedition.org/cem/11302 \\ DOI : 10.4000/cem. 11302 \\ ISSN : 1954-3093 \\ Éditeur \\ Centre d'études médiévales Saint-Germain d'Auxerre}

Édition imprimée

Date de publication : 15 août 2009

Pagination : 83-91

ISSN : 1623-5770

\section{Référence électronique}

Christian Sapin et Pierre Guibert, « Groupement de recherche européen (GdRE) du CNRS. Terres cuites architecturales et nouvelles méthodes de datation (TCA médiévales et datation) », Bulletin du centre d'études médiévales d'Auxerre / BUCEMA [En ligne], 13 | 2009, mis en ligne le 16 septembre 2009, consulté le 22 septembre 2022. URL : http://journals.openedition.org/cem/11302 ; DOI : https:// doi.org/10.4000/cem. 11302

Ce document a été généré automatiquement le 22 septembre 2022.

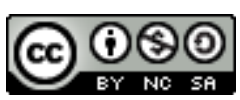

Creative Commons - Attribution - Pas d'Utilisation Commerciale - Partage dans les Mêmes Conditions 4.0 International - CC BY-NC-SA 4.0

https://creativecommons.org/licenses/by-nc-sa/4.0/ 


\title{
Groupement de recherche européen (GdRE) du CNRS. Terres cuites architecturales et nouvelles méthodes de datation (TCA médiévales et datation)
}

\author{
Christian Sapin et Pierre Guibert
}

1 En 2008 s'est achevé un chapitre du GdRE consacré aux terres cuites architecturales et aux nouvelles méthodes de datation. Nous livrons ici les premiers résultats de cette entreprise qui a obtenu récemment son renouvellement pour quatre années, 2009-2012.

2 Rappelons que ce groupe de recherche créé en 2005 correspond à la concrétisation d'un certain nombre d'actions jusqu'alors séparées pour l'étude et la datation de sites archéologiques conservés en élévation des débuts de l'époque médiévale. Il s'agissait de croiser les résultats proposés par plusieurs méthodes d'analyse pour les mêmes sites archéomagnétisme, thermoluminescence, analyse ${ }^{14} \mathrm{C}$. C'est ainsi qu'il a été mis en place un programme associant des spécialistes de l'archéométrie de laboratoires français et étrangers (cf. infra), historiens de l'art et archéologues. Ce programme réunit et structure ainsi les efforts de recherche des différents partenaires initiaux du projet en lui donnant une dimension européenne à partir des terres cuites architecturales (briques, tuiles, carrelage...). L'utilisation de l'argile comme matière première d'extraction, souvent locale, coopère d'emblée, par sa nature et sa durabilité mesurées par nos travaux, à la connaissance de savoir-faire traditionnels qui ont contribué en plusieurs régions à un développement durable du bâti. Voir l'état des résultats 2005-2008.

3 Le GdRE 2005-2008 réunit :

4 - des partenaires de laboratoires CNRS français: l'ARTeHIS - UMR 5594 Dijon; le laboratoire de médiévistique occidentale de Paris (LAMOP) - UMR 8589 ; l'Institut de recherche sur les archéomatériaux (IRAMAT-CRPAA) - UMR 5060 - université de 
Bordeaux III ; le laboratoire Géosciences-Rennes - université de Rennes I - UMR 6118 ; le laboratoire d'archéométrie et archéologie: origine, datation et technologies des matériaux - UMR 5138 - université de Lyon II ;

5 - des équipes étrangères : le Centre européen d'archéométrie de l'université de Liège (directeur Patrick Hoffsummer); le Luminescence Laboratory, Department of Archaeology, University of Durham - Grande-Bretagne (directeur Ian Bailiff) ; le Centro Universitario per le Datazioni Milano Bicocca, Laboratorio di Luminescenza, Milan Italie (chercheurs : Marco Martini et Emanuela Sibilia); le LDL 1 BBCC (Laboratorio di Datazione mediante Luminescenza e di Metodologie Fisiche applicate ai Beni Culturali), Università di Catania, Dipartimento di Fisica e Astronomia, Catane - Italie (chercheurs : Olindo Troja et Anna Gueli).

Sont également associés au projet :

- le Laboratoire des Monuments historiques ;

8 - le Service départemental d'archéologie de Maine-et-Loire, en relation avec les services de l'État concernés dans les DRAC (Service régional de l'archéologie et Monuments historiques).

9 Coordinateur principal : C. Sapin ; coordinateur adjoint : P. Guibert.

10 Les travaux du GdRE 1 (2005-2008)

11 À l'origine du programme, nous avions fait plusieurs constats, en particulier concernant l'archéologie des matériaux du bâti en plein développement depuis quelques années et sur le fait que plusieurs composants étaient l'objet d'attention et d'études par diverses équipes CNRS et universitaires - origine et taille des pierres, mortiers, enduits, fer ou plomb... -, pour certaines au sein de PCR - programme collectif de recherche du ministère de la Culture - ou de PPF - programme pluriformation multi-universitaire du MRES, «Matériaux de construction: datation, caractérisation, évolution ». Créé en 2005, le Groupe de recherche européen (GdRE) Terres cuites architecturales et nouvelles méthodes de datation (TCA médiévales et datation) est né de la nécessaire coordination entre les spécialistes de la chronologie et les archéologues du bâti. Ils se sont intéressés particulièrement pour ce projet à des édifices médiévaux antérieurs à l'an Mil comportant des éléments de terre cuite.

Pour les spécialistes du Moyen Âge, la période choisie correspond à un moment charnière dans l'évolution des techniques, où l'on redécouvre certains savoir-faire antiques et où des modes s'imposent dans certaines régions sans qu'on puisse en fixer précisément les dates. Plusieurs monuments sont emblématiques de cette époque avec des hypothèses souvent larges de datation entre le IX et le XI ${ }^{*}$ siècle. Au cours de la première phase de ce GdRE, le choix s'est porté sur un ensemble d'édifices de l'Ouest de la France, dont la plupart ont récemment fait l'objet d'étude du bâti avec des relevés et des premières analyses des mortiers. Il s'agit entre autres, de la chapelle Notre-Damesous-Terre du Mont-Saint-Michel (Manche), des églises de Saint-Martin d'Angers (Maine-et-Loire), de Saint-Philbert de Grandlieu (Loire-Atlantique), de Vieux-Pont-enAuge (Calvados), de Rugles (Eure) et du château de Mayenne (Mayenne).

Mieux dater pour mieux comprendre

Il s'agissait ici, en partant des terres cuites architecturales, et en particulier de la brique utilisée comme matériau mixte au sein de la pierre, d'avancer de nouveaux paramètres dans le champ des datations possibles, en posant les questions des 
remplois, des déplacements, des productions et en définitive de la place du composant dans une structure.

15 À partir d'édifices phares de l'Ouest de la France, nous avons montré (cf. publications) comment le couplage des études de bâti et des méthodes de datation par thermoluminescence et archéomagnétisme sur terres cuites aboutit à des informations particulièrement novatrices sur la chronologie de la production des briques, leur éventuel remploi ou sur leur production ad nove. Plus inattendu, ce couplage nous apporte des informations sur des modalités de leur fabrication.

16 Cette approche pluridisciplinaire peut, à terme, remettre en question ou au contraire confirmer les datations traditionnelles reposant, souvent, uniquement sur un texte ou un élément stylistique. Pour cela, il importait de développer les méthodes et de les croiser. L'intérêt de ce programme est moins l'écart subsistant encore dans les datations, que la combinaison des méthodes utilisées. Il s'agit en l'occurrence d'associer ${ }^{14} \mathrm{C}$, thermoluminescence, luminescence optiquement stimulée, archéomagnétisme et, dans certains cas favorables, la dendrochronologie (tableau 1).

17 La finalité pour les sciences humaines, en associant des spécialistes de chronologie et de caractérisation minérale, des historiens de l'art, et des archéologues est de proposer une autre idée de l'histoire du monument. Celui-ci ne peut être considéré comme figé dans son élaboration, car il révèle aussi les transformations sociales, religieuses et politiques de son temps. Il s'agit ainsi de mieux dater pour mieux comprendre.

18 Au-delà de la question du nombre de sites abordés, la mise en place de plusieurs disciplines d'archéométrie au rythme d'investigation et de travaux souvent lourds en laboratoire pour un même site, a entraîné un temps long pour la mise en équation des résultats et leur validation. Il existe ainsi un décalage qu'il était difficile de résorber en quatre temps. Les raisons d'une acquisition différentielle des datations sont en effet de deux ordres :

19 - la nature même des phénomènes physiques mis en jeu en datation et la complexité et la technicité des méthodes de mesure. La luminescence n'étant pas une méthode de routine, ce premier GdRE fut l'occasion de renforcer des recherches méthodologiques fondamentales pour l'obtention de résultats de qualité et exploitables;

20 - la structure des équipes impliquées : mesures de routine et laboratoire "de service " pour le radiocarbone, mesures complexes et équipes de recherche pour l'archéomagnétisme et la luminescence.

21 Nous souhaitons souligner que l'évolution structurelle de l'IRAMAT fin 2006-2007 avec l'intégration au CRP2A de l'équipe d'archéomagnétisme de Rennes et le fort développement de l'équipe luminescence de Bordeaux peuvent par ailleurs être comptés au titre des résultats institutionnels structurants majeurs du GdRE.

22 Du point de vue des archéologues et historiens de l'art, ce premier volet du GdRE a apporté des résultats tout à fait positifs en particulier pour Saint-Martin d'Angers ou le Mont-Saint-Michel, qui deviennent désormais des références pour l'avancée des techniques de construction au Xe siècle. En même temps, si certaines analyses de sites ne sont pas achevées avec des datations définitives, on sait qu'il s'agit bien de fabrication de briques médiévales et non de remplois comme cela est souvent affirmé dans les anciennes publications. Cela a des conséquences sur notre façon de considérer le renouveau de l'économie artisanale au début du Moyen Âge. Le travail commun dans les rencontres avec les diverses réunions à Paris, Poitiers, Bordeaux durant ces quatre 
années, les communications à des colloques et de publications (cf. infra), indiquent combien cette recherche, qui doit aux uns et aux autres, a profité d'une synergie entre la connaissance préalable des sites et de leur contexte en ouvrant des perspectives nouvelles. S. BÜTTNER et P. PRIGENT, «Les terres cuites architecturales comme sources d'information chronologique et technique des édifices avant l'an Mil ", in Actes du 1 er colloque francophone sur l'histoire de la construction tenu à Paris en 2008, à paraître.

"Luminescence dating of architectural ceramics from an early medieval abbey : the StPhilbert intercomparison (Loire Atlantique, France)», Radiation Measurements, sous presse.

31 C. SAPIN, "Les sources matérielles de l'architecture, apport de l'archéologue du bâtiment ", in P. HOFFSUMMER et J. ЕЕскHOUT (dir.), Matériaux de l'architecture et toits de l'Europe, 2008 (Les dossiers de l'IPW, 6), p. 19-28. construction antiques: méthodologie, essai de synthèse », Les cahiers de SaintMichel-de-Cuxa, 39 (2008), p. 223-233. Chartres. Précurseur de l'Europe Médiévale ?, Paris, 2008, p. 285-300. C. OBERLIN, "Archéologie du bâti et archéométrie au Mont-Saint-Michel, nouvelles 
approches de Notre-Dame-sous-Terre ", Archéologie médiévale, 38 (2008), p. 71-122.

E. vieILlevigne, P. guibert, A. R. zuccarello et F. Bechtel, "The Potential of Optically Stimulated Luminescence for medieval building ; a case study at Termez (Uzbekistan) », Radiation Measurements, 41 (2006), p. 991-994.

Communications à des colloques nationaux et internationaux

P. GUIBERT, E. VIEILLEVIGNE et F. BECHTEL, «Datation par luminescence : état de l'art et perspectives pour l'archéologie de l'architecture médiévale », colloque d'archéométrie GMPCA, 19-22 avril 2005, Saclay, France (oral).

P. GUIBERT, E. VIEILLEVIGNE et F. BECHTEL, « Dating architectural ceramics by luminescence methods: actual state and potentials", EMAC 05, European Meeting on Ancient Ceramics, Lyon (France), octobre 2005 (oral).

\section{En 2006 :}

41 S. blain, A. Bouvier, P. guibert, F. Bechtel, E. vieillevigne, C. SAPin et M. Baylé, «Architectural Ceramics Dating applied to Medieval Building Archaeology : the case of the church Notre-Dame-sous-Terre (Mont-Saint-Michel, France)», ISA 2006, International Symposium on Archaeometry, 2-6 mai 2006, Québec, Canada (oral).

S. blain, P. guibert, E. vieillevigne, A. Bouvier, F. Bechtel, C. SAPin et M. Baylé, «Application de la datation des Terres Cuites Architecturales à l'archéologie du bâti. Le cas de l'église de Notre-Dame-sous-Terre au Mont-Saint-Michel (Manche, France)», Colloque d'archéométrie, Aix en Provence, 18-21 avril 2007 (oral).

S. BLAIN, P. GUIBERT, E. VIEILLEVIGNE et F. BECHTEL, «Datation par luminescence des terres cuites architecturales : étude de briques de l'abbaye de Saint-Philbert de Grandlieu (44, France) », colloque d'archéométrie, Aix en Provence, 18-21 avril 2007 (poster).

S. Blain, E. vieillevigne, P. guibert, A. Bouvier, G. Pinto, F. BeChTel, C. SAPIN et M. BAYlé, "Architecture médiévale normande (églises de Vieux-Pont en Auge et d'Ouilly-leVicomte) : caractérisation physico-chimique et datation de briques par luminescence ", colloque d'archéométrie, Aix en Provence, 18-21 avril 2007 (poster).

S. BLAIN, C. SAPIN, P. GUIBERT, M. BAYLÉ et I. K. BAILLIF, «Scientific dating of Architectural Ceramics Applied to Medieval Building Archaeology : Application to the church NotreDame-sous-Terre (Mont-Saint-Michel, France)», IMC 07, colloque d'archéologie médiévale, Leeds, Grande Bretagne, 9-12 juillet 2007 (oral).

47 S. blain, P. guibert, A. bouvier, C. sapin, M. baylé, P. lanos et P. dufresne, "Ceramic building material dating applied to early medieval building archaeology in NW France », EMAC 07, European Meeting on Ancient Ceramics, Budapest (Hongrie), 24-27 octobre 2007 (oral).

P. GUIBERT, S. BLAIN, E. VIEILleVIGNE, P. LANOS, P. DUfRESNe, A. CHAUVIN, C. SAPIN, S. BÜTTNER et M. BAYLÉ, «Les terres cuites architecturales comme sources d'information chronologique et technique pour l'histoire de la construction de bâtiments préromans », Medieval Europe, Paris, septembre 2007 (oral).

\section{En 2008:}



S. O. TROJA, « Il gruppo di ricerca europeo "GdRE-TCA - terres cuites architecturales et nouvelles méthodes de datation". Presentazione dell'attività e primi risultati: datazione di laterizi dalla chiesa di S.Philbert-de-Grand-Lieu (Loire Atlantique, Francia) », colloque groupe archéométrie italien, Syracuse, février 2008 (oral).

S. Blain, D. PRigent, P. GUiBerT, C. SAPIN, A. BOUVIER et A. BEN AMARA, «Dating an early medieval church by the luminescence method, St. Martin of Angers (Pays de Loire, France) ", colloque international Archaeometry 2008, ISA 2008, Sienne, Italie, mai 2008 (poster).

P. GUiberT, I. K. BAILIFF, S. BLAin, A. M. GUeli, M. MARTini, E. SibiLiA, G. STelLA et S. O. TROJA, "Luminescence dating of architectural ceramics from an early medieval abbey : the StPhilbert investigation (Loire Atlantique, France)», colloque international Archaeometry 2008, ISA 2008, Sienne, Italie, mai 2008 (poster).

P. Guibert, C. SAPIN, S. blain, M. baylÉ, P. lanos, E. VieilleVigne, P. Dufresne, A. chauvin, S. BÜTTNER et P. PRIGENT, «Les terres cuites architecturales comme sources d'information chronologique et technique des édifices avant l'an Mil ", 1er colloque francophone sur l'histoire de la construction, Paris, juin 2008 (oral).

S. BLAIN, P. GUIBERT, I. K. BAILIFF, A. BOUVIERet M. BAYLÉ, «An intercomparison study of luminescence dating protocols and techniques applied to medieval brick samples from Normandy (France) ", 12 $2^{\text {th }}$ International Conference on Luminescence and Electron Spin Resonance Dating, 18-22 septembre 2008, Beijing China (poster).

Alliance et session de prélèvements commune en Pays d'Auge et vallée de la Risle (M. Baylé, S. Blain, T. Curling, P. Dufresne, P. Guibert et P. Lanos).

22-24 novembre 2007: Milan, université Milano Bicocca, réunion plénière du GdRE, comprenant une synthèse méthodologique sur la datation par luminescence et l'exposé de l'ensemble des résultats obtenus par le groupe. 
63 1-5 décembre 2008: université de Bordeaux, réunion de clôture du GdRE et du programme Alliance Bordeaux/Durham comprenant un séminaire sur l'apport de la chronologie physique à la datation de l'architecture et des travaux et visites sur le terrain (Notre-Dame de la Place, Saint-Seurin).

64

INDEX

Mots-clés : GDRE, TCA (Terres cuites architecturales) 